\title{
Role of lens vault in subtypes of angle closure in Iranian subjects
}

S Moghimi ${ }^{1,2,3}$, Z Vahedian', N Zandvakil'1, M Mohammdi', G Fakhraie ${ }^{1}$, N Nassiri',

AL Coleman ${ }^{2}$ and S Lin ${ }^{3}$

\begin{abstract}
Purpose The purpose of this study is to quantify anterior chamber (AC) parameters and to determine the proportion of eyes with exaggerated lens vault (LV) in different subtypes of angle closure disease using anterior segment optical coherence tomography (AS-OCT).
\end{abstract}

Patients and methods In this prospective study, 115 eyes of 115 Iranian patients with angle closure disease were included and categorized into three groups: (1) fellow eyes of acute angle closure (AAC; 40 eyes); (2) primary angle closure glaucoma (PACG; 39 eyes); and (3) primary angle closure suspect (PACS; 36 eyes). Complete ophthalmic examination including gonioscopy, A-scan biometry, and AS-OCT were performed.

Angle parameters, LV, and iris thickness (IT) were measured using AS-OCT. An exaggerated LV was defined as LV more than one-third the distance between the corneal endothelium and a line drawn to connect the nasal and temporal scleral spurs.

Results Fellow eyes of AAC had the shallower AC $(P=0.01)$, greater iris curvature (I-curve; $P=0.01)$, and higher LV $(P=0.02)$ as compared with PACS and PACG eyes. There was no statistically significant difference in the mean IT at $750 \mu \mathrm{m}$ from scleral spur among the three groups $(P=0.45)$.

Exaggerated LV was found in $67.5,35.9$, and $40 \%$ of fellow eyes of AAC, PACG, and PACS, respectively, $(P=0.008)$ with an odds ratio of $1.92(P=0.005)$ for fellow $v$ s PACG and 1.68 $(P=0.01)$ for fellow $v$ s PACS. Conclusions Exaggerated LV is highly prevalent in fellow eyes of AAC. These eyes have shallower AC depth, greater I-curve, and higher LV when compared with PACG and PACS.

Eye (2014) 28, 337-343; doi:10.1038/eye.2013.296; published online 10 January 2014
Keywords: anterior segment optical coherence tomography; angle closure; glaucoma; lens vault; iris curvature

\section{Introduction}

Angle closure disease is an anatomical disorder in which iris-trabecular contact impedes aqueous drainage through the trabecular meshwork. ${ }^{1}$ Although pupillary block and plateau iris syndrome have been proposed as the two main mechanisms in the pathogenesis of angle closure disease, other factors related to the iris, lens, and ciliary body have also been shown to have important roles. ${ }^{1,2}$ Iris, lens, and their position and relationship to each other have important roles in angle closure pathogenesis. ${ }^{1,2}$

Several ocular risk factors have been identified for angle closure disease such as short axial length, shallow anterior chamber (AC), thick peripheral iris, and a thick, anteriorly positioned lens. ${ }^{1,3-5}$ With the advent of anterior segment optical coherence tomography (ASOCT), researchers can capture the entire AS in a single image and assess angle, iris, and lens parameters more precisely. Lens vault (LV), defined as the perpendicular distance between the anterior lens pole and the horizontal line joining the temporal and nasal scleral spurs, is one of the novel parameters that can be measured with AS-OCT and has been associated with angle closure. ${ }^{6,7}$ Iris curvature (I-curve) has been proposed to be an indicator of pupillary block. ${ }^{8,9}$ As I-curve is reported to be only moderately correlated with increased LV, pupillary block may not be the only mechanism by which increased LV causes angle closure., 90 In fact, there are some cases with exaggerated $\mathrm{LV}$ in which the iris appears to drape the anterior surface of the lens, giving rise to a 'volcano-like configuration' without an increase
${ }^{1}$ Eye Research Center, Farabi Eye Hospital, Tehran University of Medical Science, Tehran, Iran

${ }^{2}$ Glaucoma Division, Jules Stein Eye Institute, David Geffen School of Medicine, University of California at Los Angeles, Los Angeles, CA, USA

${ }^{3}$ Koret Vision Center, University of California, San Francisco Medical School, San Francisco, CA, USA

Correspondence:

S Moghimi, Eye Research Center, Farabi Eye Hospital, Tehran University of Medical Science, Quazvin Square, Tehran, 13364, Iran. Tel: +98 21 55416134; Fax: +98 2155416134 . E-mail: sasanimii@ yahoo.com

Received: 25 August 2013 Accepted in revised form: 11 November 2013 Published online: 10 January 2014

The article has been presented as a poster at the 5th World Glaucoma Congress 2013-Vancouver, Canada. 
in I-curve. ${ }^{2,11}$ Exaggerated LV has been defined quantitatively as LV more than one-third the distance between the corneal endothelium and a line drawn to connect the nasal and temporal scleral spurs. ${ }^{12}$

Angle closure disease is classified into different subtypes including primary angle closure suspect (PACS), acute angle closure (AAC), and primary angle closure glaucoma (PACG). ${ }^{11,13-15}$ Quantitative evaluation of the AS in these eyes might be helpful in explaining the pathogenesis of angle closure. In this study, different subtypes of angle closure disease including fellow eyes of AAC, PACG, and PACS were evaluated and prevalence of exaggerated LV was reported. AS-OCT and A-scan biometry were used to measure ocular biometric parameters, including the iris thickness (IT), I-curve, lens characteristics, and LV.

\section{Patients and methods}

\section{Patients}

In this cross-sectional study, 154 eyes (154 patients) with at least one eye with AAC, PACS status, and PACG, as defined below, were consecutively recruited from the Glaucoma Clinic - a tertiary care center-of the Farabi Eye Hospital before a laser iridotomy. All recruited subjects were drawn from the Iranian race. The Ethics Committee at the Farabi Eye Hospital approved the study protocol. All patients provided written informed consent forms in accordance with the Declaration of Helsinki. Only the right eyes of patients were included for analysis in this study. If the left eye was the only affected one, then the left eye was included. Eyes with a history of pilocarpine usage, trauma, uveitis, ocular laser, and/or surgical procedures (eg, laser peripheral iridotomy, LPI) were excluded. In addition, eyes with iris or angle neovascularization, pseudoexfoliation, any kind of secondary angle closure, or any iris or corneal abnormalities were excluded. None of the patient had taken any miotic or mydriatic medications.

After excluding eyes with poor AS-OCT image quality (28 eyes), pseudoexfoliation (4 eyes), prior LPI (3 eyes), and secondary angle closure (2 eyes), a total of 115 eyes (115 patients) were classified into one of the following three groups: (1) the fellow eye of AAC (40 eyes),

(2) PACS (36 eyes), and (3) PACG (39 eyes).

(1) Fellow eyes of AAC: AAC was defined as: (a) at least two of the symptoms of an acute episode of intraocular pressure (IOP) rise that are ocular pain or headache, nausea and/or vomiting, decreased vision, and rainbow-colored halos around lights; (b) IOP at presentation of at least $30 \mathrm{~mm} \mathrm{Hg}$ with Goldmann applanation tonometry; (c) examination findings such as conjunctival injection, corneal epithelial edema, fixed mid-dilated pupil and shallow AC; and (d) shallow AC and narrow angle in the other eye that was defined as our first group (fellow eye).

(2) PACG eyes had chronically elevated IOP above $21 \mathrm{~mm} \mathrm{Hg}$ (before treatment) along with glaucomatous optic neuropathy, shallow AC, and iridotrabecular contact in at least three quadrants on gonioscopy along with a variable amount of peripheral anterior synechiae (PAS).

(3) PACS eyes were classified based on the posterior trabecular meshwork not being visible in at least three quadrants without PAS or any evidence of glaucomatous optic nerve or visual field damage, and IOP $\leq 21 \mathrm{~mm} \mathrm{Hg}$ without medication.

There was no history or sign of previous acute glaucoma attack in all the three groups.

\section{Examinations}

Slit lamp examination of the AS, Goldmann applanation tonometry, and gonioscopy in dark conditions (with and without indentation) were conducted in all patients. A glaucoma specialist (SM) performed indentation gonioscopy using a Zeiss-style four-mirror goniolens (Model G-4, Volk Optical, Mentor, OH, USA) with a narrow 1-mm beam of light.

A-scan biometry (Echoscan, model U3300, Nidek, Tokyo, Japan) was used to measure axial length, lens thickness (LT), and AC depth (ACD). Five readings were taken for each eye. After omitting the highest and lowest values, the mean of the other three readings was used for analysis

All subjects underwent static automated white-onwhite threshold perimetry (program 24-2, Swedish Interactive Threshold Algorithm standard, model 750, Humphrey Field Analyzer, Humphrey Instruments, Dublin, CA, USA). Visual fields were read by a glaucoma specialist (MM) who was masked to the clinical data. The reliability of visual field tests was defined as fixation loss and false positive and negative response rates of $<15 \%$. If the visual field was deemed unreliable by the grader, then it was repeated.

\section{Anterior segment optical coherence tomography}

AS-OCT (Visante OCT; Carl Zeiss Meditec, Dublin, CA, USA) was performed for all the patients in dark lighting (in a room without windows, with door closed, and the only lighting with AS-OCT screen). Scans were centered on the pupil and were obtained along the horizontal and the vertical axes using the enhanced AS single protocol. Two images were captured for each axis and the one with 
higher quality was chosen for analysis. The brightness and contrast of each image were adjusted so that the scleral spurs could be detected as clear as possible. Two experienced ophthalmologists (SM and ZV) determined scleral spur location in each image. The principal investigator (SM) validated all the images for quality and scleral spur location.

Because the superior and inferior angle images usually do not have the optimal quality and are not reproducible, ${ }^{16}$ only the temporal and nasal angle parameters were used for analysis. The following parameters were measured using the tools provided by the machine (Visante Software Version 2.0.1.88, Carl Zeiss Meditec).

1. Angle opening distance at $750 \mu \mathrm{m}$ from scleral spur (the distance between the posterior corneal surface and the anterior iris surface on a line perpendicular to the trabecular meshwork, $500 \mu \mathrm{m}$ from the scleral spur). $2,5,17,18$

2. Trabeculo-iris space area at $750 \mu \mathrm{m}$ from scleral spur (the surface area of a trapezoid with the following boundaries: anteriorly, the angle opening distance at $500 \mu \mathrm{m}$ from scleral spur; posteriorly, a line drawn from the scleral spur perpendicular to the plane of the inner scleral wall to the iris; superiorly, the inner corneoscleral wall; and inferiorly, the iris surface). ${ }^{18}$

3. AC angle: The trabecular-iris angle measured with the apex in the iris recess and the arms of the angle passing through a point on the trabecular meshwork at $500 \mu \mathrm{m}$ from the scleral spur and the point on the iris perpendicularly opposite. ${ }^{5}$

4. LV, which is the perpendicular distance from the anterior lens surface to the horizontal line connecting the two scleral spurs, was measured by the chamber tool of the Visante OCT. When the anterior pole of the lens was located anterior to the scleral spur line, LV was positive, and when it was posterior to the line, LV was reported as negative. ${ }^{6}$
5. I-curve was another parameter that was measured by drawing a line between the most peripheral and most central points of the iris pigment epithelium and then measuring the largest perpendicular distance from this line to the iris pigment epithelium. ${ }^{19}$

6. IT was measured at $750 \mu \mathrm{m}$ (IT750) anterior to the scleral spur.

The mean of the nasal and temporal angle opening distance at $750 \mu \mathrm{m}$ from scleral spur, trabeculo-iris space area at $750 \mu \mathrm{m}$ from scleral spur, AC angle, I-curve, and IT were labeled as AOD750, TISA750, ACA, I-curve, and IT750, respectively. Exaggerated LV has been defined quantitatively as LV that is more than one-third the distance between the corneal endothelium and a line drawn to connect the nasal and temporal scleral spurs (Figure 1).

\section{Statistical analysis}

Statistical analysis was performed using SPSS software version 17 (SPSS, Inc., Chicago, IL, USA). Parametric variables were analyzed using ANOVA and post hoc Tukey tests. Analysis of nonparametric variables was done by the Kruskal-Wallis test. $\chi^{2}$ testing was used for analysis of qualitative variables. Pearson and Spearman correlation coefficients were calculated between LV and parametric and nonparametric variables, respectively. $P$-value of $<0.05$ was considered as statistically significant.

\section{Results}

The demographic and clinical examination data of the three groups are summarized in Table 1. There was no difference in age and gender among these groups. As would be expected, the IOP was significantly greater in PACG eyes than the other groups, even with the use of

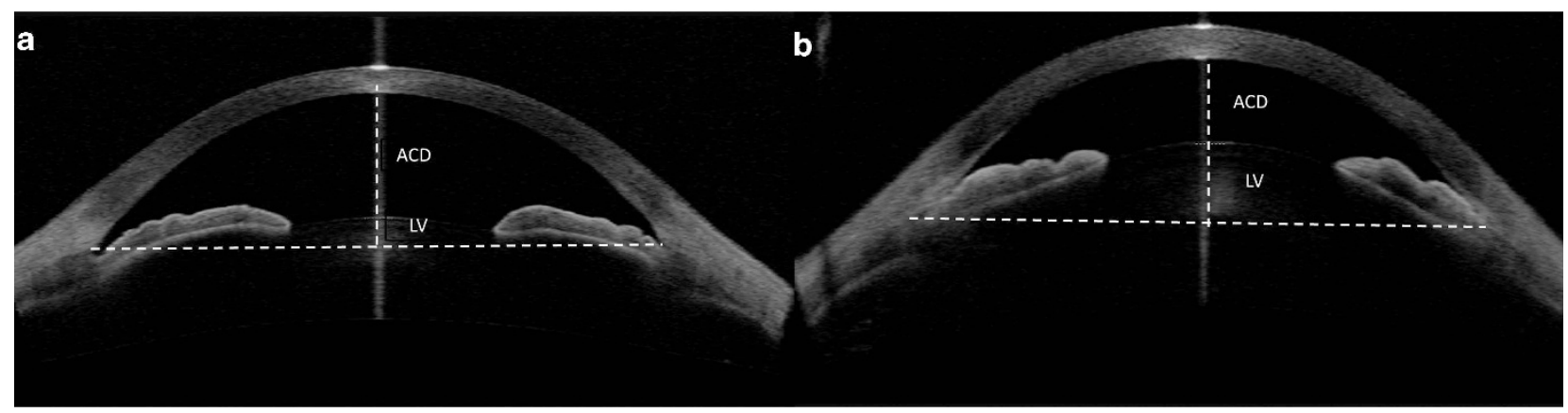

Figure 1 (a) Anterior segment optical coherence tomographic scan showing LV and ACD. (b) AS-OCT scan showing an eye with exaggerated LV in which the LV is more than one-third the distance between the corneal endothelium and a line drawn to connect the nasal and temporal scleral spurs. 
Table 1 Comparison of demographic and clinical examination data in angle closure subtypes

\begin{tabular}{lcccc}
\hline & Fellow eyes & PACG & PACS & P-value \\
\hline No. of eyes & 40 & 39 & 36 & - \\
Age (years), mean \pm SD & $59.4 \pm 9.2$ & $60.7 \pm 9.0$ & $60.2 \pm 8.1$ & $27 / 9$ \\
Female/male & $31 / 9$ & $20 / 19$ & $15.8 \pm 2.6$ & $<.09$ \\
IOP (mm Hg), mean \pm SD & $12.7 \pm 3.2$ & $20.8 \pm 7.4$ & $1.7 \pm 1.3$ & $0.0 \pm 0.0$ \\
Medication number, mean \pm SD & $1.0 \pm 0.7$ & $0.68 \pm 0.21$ & $0.29 \pm 0.09$ & $<0.001$ \\
C/D ratio, mean \pm SD & $0.30 \pm 0.11$ & $0.54 \pm 0.59$ & $0.64 \pm 0.57$ & 0.31 \\
Gonioscopy, mean \pm SD & $0.44 \pm 0.55$ & $184.0 \pm 146.9$ & $0.0 \pm 0.0$ & $<0.001$ \\
PAS (degrees), mean \pm SD & $13.1 \pm 32.6$ & $2.7 \pm 0.9$ & $2.6 \pm 0.9$ & 0.32 \\
Lens nucleus opacity (per LOCS III), mean \pm SD & $2.5 \pm 0.7$ & &
\end{tabular}

Abbreviations: C/D, cup-to-disc; IOP, intraocular pressure; LOCS III, lens opacities classification system III; PACG, primary angle closure glaucoma; PACS, primary angle closure suspect; PAS, peripheral anterior synechiae.

anti-glaucoma medications $(P<0.001)$. These eyes also had a larger cup-to-disc ratio $(P<0.001)$ (Table 1$)$. Agreement between two observers on the position of the scleral spur on AS-OCT was good (0 kappa coefficient $=0.87$ ).

\section{Angle status}

PACG eyes had 184.0 \pm 146.9 degrees of PAS that was significantly more compared with the other two groups $(P<0.001)$. Fifteen percent (6 eyes) of our fellow eye group and 69\% (27 eyes) of the PACG group had PAS in their angles. Gonioscopically, the AC angle was narrowest in the fellow eyes, followed by eyes with PACG and PACS. However, the difference did not reach statistical significance. ACA, AOD750, and TISA750 were significantly less in fellow eyes and the PACG group compared with the PACS group (Table 2; $P<0.001$ for all).

\section{$A C D$, lens status, and iris parameters}

The eyes with PACS had similar ACD as PACG eyes ( $2.53 \pm 0.28$ vs $2.49 \pm 0.25$, respectively; $P=0.97$ ), but had deeper AC than fellow eyes in the AAC group (2.36 $\pm 0.21 ; P<0.04 ;$ Table 2$)$. Lens nucleus opacification was similar among the three angle closure groups (Table 1). There was also no statistically significant difference in LT among the three groups $(P=0.48)$. The LV was highest $(977.00 \pm 192.32 \mu \mathrm{m})$ in fellow eyes followed by PACS eyes; the lowest LV was observed in PACG eyes $(851.28 \pm 186.65 \mu \mathrm{m} ; P<0.001)$. There was no statistically significant difference in the mean IT750 among the three groups $(P=0.45)$; however, I-curve was greater in fellow eyes $(0.29 \pm 0.09)$, followed by PACS $(0.27 \pm 0.08)$ and PACG eyes $(0.27 \pm 0.08)(P=0.01)$.

Exaggerated LV was found in $27(67.5 \%), 14(35.9 \%)$, and $16(40 \%)$ of fellow eyes of AAC, PACG, and PACS,
Table 2 Angle, anterior chamber, and lens parameters of subtypes of angle closure and normal control eyes measured by anterior segment optical coherence tomography or A-scan ultrasound

\begin{tabular}{lcccc}
\hline Parameters & Fellow eyes & PACG & PACS & P-value \\
\hline AOD750 $(\mathrm{mm})$ & $0.074 \pm 0.043$ & $0.113 \pm 0.083$ & $0.146 \pm 0.088$ & $<0.001$ \\
TISA750 $\left(\mathrm{mm}^{2}\right)$ & $0.036 \pm 0.022$ & $0.047 \pm 0.038$ & $0.082 \pm 0.040$ & $<0.001$ \\
ACA $\left({ }^{\circ}\right)$ & $3.19 \pm 2.73$ & $5.00 \pm 4.81$ & $8.96 \pm 5.08$ & $<0.001$ \\
LT $(\mathrm{mm})$ & $4.96 \pm 0.32$ & $4.86 \pm 0.40$ & $4.92 \pm 0.30$ & 0.48 \\
ACD $(\mathrm{mm})$ & $2.36 \pm 0.21$ & $2.49 \pm 0.25$ & $2.53 \pm 0.28$ & 0.01 \\
AL $(\mathrm{mm})$ & $21.69 \pm 1.13$ & $22.48 \pm 0.82$ & $21.97 \pm 0.73$ & 0.001 \\
LV $(\mu \mathrm{m})$ & $977.00 \pm 192.32$ & $851.28 \pm 186.65$ & $890.25 \pm 221.30$ & 0.02 \\
I-curve $(\mathrm{mm})$ & $0.29 \pm 0.09$ & $0.22 \pm 0.11$ & $0.27 \pm 0.08$ & 0.01 \\
IT750 $(\mathrm{mm})$ & $0.47 \pm 0.09$ & $0.44 \pm 0.08$ & $0.46 \pm 0.12$ & 0.45 \\
\hline
\end{tabular}

Abbreviations: ACA, average of nasal and temporal anterior chamber angle; $\mathrm{ACD}$, anterior chamber depth; $\mathrm{AL}$, axial length; $\mathrm{AOD750}$, average of nasal and temporal angle opening distance at $750 \mu \mathrm{m}$ from scleral spur; I-curve, average of nasal and temporal iris curvature; IT750, average of nasal and temporal iris thickness at750 $\mu \mathrm{m}$ from scleral spur; LT, lens thickness; LV, lens vault; PACG: primary angle closure glaucoma; PACS: primary angle closure suspect; TISA750, average of nasal and temporal trabeculo-iris space area at $750 \mu \mathrm{m}$ from scleral spur.

respectively $(P=0.008)$. The odds ratio for fellow $v s$ PACG was $1.92(P=0.005)$ and for fellow vs PACS was $1.68(P=0.01)$ for having exaggerated LV. The eyes with exaggerated LV had greater I-curve $(0.30 \pm 0.08 \mathrm{~mm})$ compared with the other group $(0.22 \pm 0.10 ; P<0.001)$. Although IT was thinner in the exaggerated LV group $(0.43 \pm 0.08$ vs $0.47 \pm 0.11)$, the difference between two groups did not reach statistical significance $(P=0.15)$.

\section{Discussion}

In this cross-sectional study, exaggerated LV was found in two-thirds of fellow eyes of AAC, whereas only onethird of PACS or PACG eyes demonstrated this status. The fellow eyes of AAC had the greatest LV and I-curve, although the biometric parameters of PACS and PACG 
were not significantly different from each other. This is consistent with recent findings from another study on the subtypes of angle closure in Asian patients using AS-OCT. ${ }^{13}$

The biometric characteristics of eyes with narrow angle have been studied extensively, particularly in Asian populations. These eyes usually have shorter axial length, shallower AC, smaller corneal diameter, and a thicker and more anteriorly positioned lens than that in open angle eyes. ${ }^{3-5,20,21}$ A shallow AC is the most important and consistent biometric feature predisposing to AAC in different ethnicities ${ }^{5,22}$ including Iranians. It has been shown that up to $50 \%$ of the fellow eyes of AAC patients will have an attack within 5 years if left untreated, ${ }^{23,24}$ and are considered to be in the pre-attack stage. ${ }^{13,25}$

Friedman et $a l^{25}$ reported that the fellow eyes of patients with AAC have a mean ACD that was $0.63 \mathrm{~mm}$ shallower than normal controls. We found that the mean ACD in AAC eyes was $0.17 \mathrm{~mm}$ less than that in PACS eyes. The significant difference between this group and the PACS group supports the importance of the ACD in development of AAC. For this reason, some investigators proposed that the shallower the ACD, the higher the risk of an acute attack. ${ }^{13,26}$

LV is a newly introduced parameter that represents the height of the lens anterior to the scleral spur plane, and has been shown to be a significant predictor of angle closure disease. ${ }^{6}$ Our results show that the fellow eyes of AAC eyes had greater LV compared with other groups especially PACG eyes. The greater amount of lens protruding anteriorly may lead to a narrower angle and predispose the eye to an acute attack of angle closure. The greater the LV, the more the iris is pushed anteriorly. This increases iridolenticular contact and lengthens and narrows the iris-lens channel, thereby causing pupillary block. ${ }^{1}$

In the present study, the differences in LT between subtypes of angle closure disease were not significant. This is in agreement with the study by Mimiwati et al ${ }^{15}$ in which they similarly did not report any difference in LT between AAC, subacute, and chronic angle closure glaucoma patients. This might suggest that LT is not an important pathogenic factor for developing AAC glaucoma, and the amount of its anterior protrusion is more important. ${ }^{3}$

Shabana et $a l^{2}$ reported a category of eyes with exaggerated LV in which the iris appears to drape the anterior surface of the lens, giving rise to a 'volcano-like configuration' without any increase in I-curve. Quantitatively, we found that exaggerated LV is significantly more prevalent in fellow eyes of AAC compared with other groups. IT was nonsignificantly thinner in our cases with exaggerated LV compared with the other group, but I-curve was significantly greater. It seems that higher LV predisposes the eye to angle closure through multiple mechanisms. ${ }^{1,6}$ In addition to pupil block, pushing the iris directly and crowding the angle in these eyes might be responsible for narrowing of the angle in these eyes.

Consistent with our study, using agglomerative hierarchical clustering method, Nongpiur et al ${ }^{27}$ categorized PACS eyes into three subgroups. One subgroup was characterized by a large LV and a shallow ACD; a second subgroup was characterized by a large iris area; and the third subgroup was characterized by elements of both the first and the second subgroups. They proposed that the predominant mechanism for the first subgroup was a large LV even though iris area was smaller than that seen in the second subgroup, suggesting more of a pushing mechanism.

Although some population-based studies demonstrated that IT is independently associated with narrow angles, particularly in women and older subjects, ${ }^{28}$ few studies evaluate that in subtypes of angle closure. A recent finding shows that AAC eyes have thicker IT compared with other subgroups of angle closure glaucoma. ${ }^{9}$ However, in the study by Sihota et $a l^{22}$, AAC eyes had similar IT as PACG eyes, but it was less than that in control eyes. Henzan et al ${ }^{29}$ did not find any difference in IT among eyes with PAC, PACS, and controls. Similarly, we did not find any difference between fellow eyes of patients with AAC, PACS, and PACG. The discrepant findings may be because of either the interval between acute attack and the capture of ASOCT images or the differences in IT between different populations.

It has been shown that a steeper iris is an independent predictor of AC angle widening after LPI in angle closure patients. ${ }^{30}$ In our series of patients, PACG eyes had the lowest I-curve among the groups. High I-curve in PACS and fellow eyes of AAC may be one of the reason why LPI is so effective in these eyes. ${ }^{23,24,31}$

The results of our study should be interpreted with its limitations in mind. Because patients were of Iranian descent, the results of this study may not be applicable to other ethnic groups. Another limitation was that we did not perform ultrasound biomicroscopy in our patients to diagnose plateau iris configuration more precisely.

In conclusion, we found that fellow eyes of AAC had the narrowest angles, and the highest LV and greatest I-curves, in comparison with PACS and PACG eyes. Exaggerated LV was more prevalent in fellow eyes of AAC among the three groups. These findings may be relevant for understanding angle-closure pathogenesis and management. 


\section{Summary}

\section{What was known before}

- LV is a factor related to AS crowding. Exaggerated LV is a category of angle closure eyes in AS-OCT related to lensinduced angle closure mechanism.

\section{What this study adds}

- Fellow eyes of AAC had the shallowest AC, greater I-curve, and LV than that in PACS and CACG eyes. Exaggerated LV was more prevalent in fellow eyes of AAC compared with PACG and PACS.

\section{Conflict of interest}

The authors declare no conflict of interest.

\section{Acknowledgements}

We would like to thank Dr Meera Ramanathan (Glaucoma Division, Jules Stein Eye Institute, University of California at Los Angeles) for her assistance in the preparation of the manuscript.

\section{References}

1 Tarongoy P, Ho CL, Walton DS. Angle-closure glaucoma: the role of the lens in the pathogenesis, prevention, and treatment. Survey Ophthalmol 2009; 54(2): 211-225.

2 Shabana N, Aquino MCD, See J, Ce Z, Tan AM, Nolan WP et al. Quantitative evaluation of anterior chamber parameters using anterior segment optical coherence tomography in primary angle closure mechanisms. Clin Exp Ophthalmol 2012; 40(8): 792-801.

3 George R, Paul P, Baskaran M, Ramesh SV, Raju P, Arvind H et al. Ocular biometry in occludable angles and angle closure glaucoma: a population based survey. $\mathrm{Br} \mathrm{J}$ Ophthalmol 2003; 87(4): 399-402.

4 Lavanya R, Wong TY, Friedman DS, Aung HT, Alfred T, Gao $\mathrm{H}$ et al. Determinants of angle closure in older Singaporeans. Arch Ophthalmol 2008; 126(5): 686-691.

5 Marchini G, Pagliarusco A, Toscano A, Tosi R, Brunelli C, Bonomi L. Ultrasound biomicroscopic and conventional ultrasonographic study of ocular dimensions in primary angle-closure glaucoma. Ophthalmology 1998; 105(11): 2091-2098.

6 Nongpiur ME, He M, Amerasinghe N, Friedman DS, Tay WT, Baskaran $\mathrm{M}$ et al. Lens vault, thickness, and position in Chinese subjects with angle closure. Ophthalmology 2011; 118(3): 474-479.

7 Nongpiur ME, Sakata LM, Friedman DS, Mingguang H, Chan YH, Lavanya $\mathrm{R}$ et al. Novel association of smaller anterior chamber width with angle closure in Singaporeans. Ophthalmology 2010; 117(10): 1967-1973.

8 Nonaka A, Iwawaki T, Kikuchi M, Fujihara M, Nishida A, Kurimoto Y. Quantitative evaluation of iris convexity in primary angle closure. Am J Ophthalmol 2007; 143(4): 695-697.

9 Guzman CP, Gong T, Nongpiur ME, Perera SA, How AC, Lee HK et al. Anterior segment optical coherence tomography parameters in subtypes of primary angle closure. Invest Ophthalmol Vis Sci 2013; 54(8): 5281-5286.

10 Moghimi S, Vahedian Z, Fakhraie G, Ghaffari R, Eslami Y, Jabarvand $\mathrm{M}$ et al. Ocular biometry in the subtypes of angle closure: an Anterior Segment Optical Coherence Tomography Study. Am J Ophthalmol 2012; 155: 664-673.

11 Foster PJ, Buhrmann R, Quigley HA, Johnson GJ. The definition and classification of glaucoma in prevalence surveys. Br J Ophthalmol 2002; 86(2): 238-242.

12 Sihota R, Vashisht P, Sharma A, Chakraborty S, Gupta V, Pandey RM. Anterior segment optical coherence tomography characteristics in an Asian population. J Glaucoma 2012; 21(3): 180-185.

13 Lan YW, Hsieh JW, Hung PT. Ocular biometry in acute and chronic angle-closure glaucoma. Ophthalmologica 2007; 221(6): 388-394.

14 Mérula RV, Cronemberger S, Diniz Filho A, Calixto N. New comparative ultrasound biomicroscopic findings between fellow eyes of acute angle closure and glaucomatous eyes with narrow angle. Arq Bras Oftalmol 2008; 71(6): 793-798.

15 Mimiwati Z, Fathilah J. Ocular biometry in the subtypes of primary angle closure glaucoma in University Malaya Medical Centre. Med J Malaysia 2001; 56(3): 341-349.

16 Nolan WP, See JL, Aung T, Friedman DS, Chan YH, Smith $\mathrm{SD}$ et al. Changes in angle configuration after phacoemulsification measured by anterior segment optical coherence tomography. J Glaucoma 2008; 17(6): 455-459.

17 Radhakrishnan S, Goldsmith J, Huang D, Westphal V, Dueker DK, Rollins AM et al. Comparison of optical coherence tomography and ultrasound biomicroscopy for detection of narrow anterior chamber angles. Arch Ophthalmol 2005; 123(8): 1053-1059.

18 Radhakrishnan S, Huang D, Smith SD. Optical coherence tomography imaging of the anterior chamber angle. Ophthalmol Clin North Am 2005; 18(3): 375-381.

19 Dorairaj S, Oliveira C, Fose AK, Liebmann JM, Tello C, Barocas $\mathrm{VH}$ et al. Accommodation-induced changes in iris curvature. Exp Eye Res 2008; 86(2): 220-225.

20 Casson RJ. Anterior chamber depth and primary angle-closure glaucoma: an evolutionary perspective. Clin Exp Ophthalmol 2008; 36(1): 70-77.

21 Tomlinson A, Leighton D. Ocular dimensions in the heredity of angle-closure glaucoma. Br J Ophthalmol 1973; 57(7): 475-486.

22 Sihota R, Dada T, Gupta R, Lakshminarayan P, Pandey RM. Ultrasound biomicroscopy in the subtypes of primary angle closure glaucoma. J Glaucoma 2005; 14(5): 387-391.

23 Bain $W$. The fellow eye in acute closed-angle glaucoma. Br J Ophthalmol 1957; 41(4): 193-199.

24 Lowe RF. Acute angle-closure glaucoma: the second eye: an analysis of 200 cases. Br J Ophthalmol 1962; 46(11): 641-650.

25 Friedman DS, Gazzard G, Foster P, Devereux J, Broman A, Quigley $\mathrm{H}$ et al. Ultrasonographic biomicroscopy, Scheimpflug photography, and novel provocative tests in contralateral eyes of Chinese patients initially seen with acute angle closure. Arch Ophthalmol 2003; 121(5): 633-642.

26 Ang L, Aung T, Chew P. Acute primary angle closure in an Asian population: long-term outcome of the fellow eye after prophylactic laser peripheral iridotomy. Ophthalmology 2000; 107(11): 2092-2096.

27 Nongpiur ME, Gong T, Lee HK, Perera SA, Cheng L, Foo L-L et al. Subgrouping of primary angle-closure suspects based on anterior segment optical coherence tomography parameters. Ophthalmology 2013; 120(12): 2525-2531. 
28 Whang B, Sakata LM, Friedman DS, Chan YH, He M, Lavanya $\mathrm{R}$ et al. Quantitative iris parameters and association with narrow angles. Ophthalmology 2010; 117(1): 11-17.

29 Henzan IM, Tomidokoro A, Uejo C, Sakai H, Sawaguchi S, Iwase A et al. Comparison of ultrasound biomicroscopic configurations among primary angle closure, its suspects, and nonoccludable angles: the Kumejima Study. Am J Ophthalmol 2011; 151(6): 1065-1073.
30 Huang G, Gonzalez E, Lee R, Osmonavic S, Leeungurasatien $\mathrm{T}, \mathrm{He} \mathrm{M}$ et al. Anatomic predictors for anterior chamber angle opening after laser peripheral iridotomy in narrow angle eyes. Curr Eye Res 2012; 37(7): 575-582.

31 Ang GS, Wells AP. Factors influencing laser peripheral iridotomy outcomes in white eyes: an anterior segment optical coherence tomography study. J Glaucoma 2011; 20(9): 577-583. 